\title{
Ventajas fundamentadas y consideraciones del sistema de tuberías de polipropileno beta nucleado frente al acero inoxidable
}

Pedro Angel Toribio Pando ${ }^{1}$

Pablo Santa Cruz Roldán ${ }^{2}$ Alberto Landauro Abanto ${ }^{3}$

\begin{abstract}
RESUMEN
El polímero es un material termoplástico que puede cristalizarse en las siguientes formas: a (monoclíica), $\beta$ (trigonal) y $\Upsilon$ (ortorrómbica); estas formas poseen características que aportan propiedades fisicoquímicas específicas en una misma formulación. La forma cristalina a se encuentra en mayor proporción y es la más estable y sencilla de producir. Sin embargo, en las materias primas de las tuberías de polipropileno beta nucleado y monocapa RP (Resistente a la presión) y tuberías de fibra de polipropileno RP, se realizan los procesos necesarios para producir estructuras $\beta$ en posiciones donde lo más sencillo sería disponer de cristales $a$, ya que este proceso favorece la formación de una estructura cristalina hexagonal estable, fina y una distribución de tamaño homogénea. Estos atributos derivan en una optimización de las prestaciones mecánicas del sistema, en la que una mezcla de estas formas (a y $\beta$ ) va a proporcionar una propiedad termomecánica muy óptima.
\end{abstract}

Palabras clave: PP-R (Polipropileno); trasiego: biocida; austeníticos; biocapa.

\section{INTRODUCCIÓN}

El inventor estadounidense John Hyatt diseñó el celuloide, que es un material plástico y cuya composición consta de una disolución constituida por carbohidrato proveniente de las plantas, celulosa y una mezcla de alcohol etílico y alcanfor; asimismo, recibe el nombre de "material termoplástico" debido a su propiedad de ser moldeado y suavizado cuando se expone al calor (Concha, 2014).

Posteriormente llegaría en 1907 la baquelita, un invento de Leo Baekeland, que fue calificada como el primer plástico termoestable o termofijo, es decir, que es moldeable cuando se funde. Para 1930, en Inglaterra se fabricó por primera vez el polietileno (PE), un termoplástico resultado de la polimerización del gas etileno sometido a presión y calor. Alrededor de 1950 se creó de manera similar el polipropileno, resultado del mismo proceso aplicado al propileno. Actualmente este termoplástico parcialmente cristalino es clasificado como una poliolefina y su uso es muy recurrente para múltiples aplicaciones (Concha, 2014). Según sus propiedades, las aplicaciones industriales están más asociadas al uso en el transporte de ácidos y bases, incluso a altas temperaturas (Concha, 2014).

El sistema de tuberías de polipropileno beta nucleado es una de las mejores elecciones para la fabricación de tuberías destinadas al trasiego de fluidos como, por ejemplo, el agua potable. El rango de trabajo en cuanto a presión y temperatura del PP-R (Polipropileno Copolímero Random) del sistema de tuberías de polipropileno beta nucleado es totalmente compatible con los

1 Ingeniero mecánico electricista y Doctor en Ingeniería Eléctrica por la Universidad Nacional de Ingeniería (Lima, Perú). Actualmente, es docente universitario en la Universidad Nacional de Ingeniería (Lima, Perú)

ORCID: https://orcid.org/0000-0002-1755-6473

Autor de correspondencia: ptoribio.consultor.ing@gmail.com

2 Licenciado en Química General por la Universidade da Coruña (A Coruña, España). Actualmente, trabaja como ingeniero en la Empresa ITALSAN (Madrid, España). ORCID: https://orcid.org/0000-0002-3153-6284

E-mail: psantacruz@mundoecco.com

3 Ingeniero metalurgista y Doctor en Administración por la Universidad Nacional de Ingeniería (Lima, Perú). Actualmente, es director de la Escuela de Ingeniería de Metalurgia y director de la Escuela de Posgrado de la Facultad de Ingeniería Geológica Minera y Metalurgia de la Universidad Nacional de Ingeniería (Lima, Perú). ORCID: https://orcid.org/0000-0003-3748-120X E-mail: albertolandauro@yahoo.es 
tratamientos para prevenir la contaminación por Legionella durante el mantenimiento de las tuberías como, por ejemplo, el choque térmico.

Esto significa que la prevención de la legionelosis se encuentra bajo constante evaluación y, en consecuencia, presenta cambios periódicos. Este tema es más relevante en países donde existe relación entre el desarrollo de las enfermedades y la industria del país. Dicha relación no es observable en otros países, ya sea por la falta de análisis o porque la contaminación por Legionella no es frecuente. En efecto, el control continuo, las nuevas estrategias y la evaluación resultan en una buena prevención de contraer enfermedades como la legionelosis. Es un hecho que la conservación de los equipos y las condiciones de su entorno son importantes; sin embargo, en el ámbito urbano, el mal es causado por malos diseños de la edificación, inadecuada construcción y descuido del control de la calidad del agua de las instalaciones (Gea, Mezones, y Haro, 2012).

En suma, el PP-R del sistema de tuberías de polipropileno beta nucleado cumple con el Decreto Supremo N. ${ }^{\circ}$ 031-2010 (2010), en donde se imponen las normativas acerca de la administración de la calidad de agua con fines de consumo humano, con el objetivo de asegurar su inocuidad, evitar riesgos para la salud humana y así mantener la salubridad en la población.

El uso de $\mathrm{ClO}_{2}$ no es recomendable para la desinfección del agua de consumo.

Históricamente, los metales han sido siempre la primera opción en la elección de materiales para fontanería. Sin embargo, tal y como se ha visto, los polímeros técnicos han ido sustituyéndolos. A diferencia de lo que su propio nombre especifica, el acero inoxidable no es inoxidable. Lo que permite que tenga este nombre es que, en comparación con el acero normal, puede soportar mucho más tiempo ante unas mismas condiciones de trabajo antes de mostrar signos de fatiga. Sin embargo, no todos los aceros inoxidables tienen las mismas propiedades ni, por supuesto, calidades.

Los aceros inoxidables austeníticos son los más comunes, de los cuales, la serie 300 es el subgrupo más grande y, dentro de este, el AISI 304, seguido por el AISI 316, son sus dos referencias más comunes, cuyo alto contenido en cromo y níquel aumentan su resistencia a la corrosión. Además, el manganeso, fósforo, sulfuro y silicio también están integrados a su composición química, que de forma general es hierro (Goodfellow, s.f.a; Goodfellow, s.f.b). Las diferencias entre ambas aleaciones pueden ser considerables, dependiendo de la instalación, siendo la más notable el contenido de molibdeno en el AISI 316, que mejora la resistencia a la corrosión con respecto al AISI 304 en ambientes salinos o expuestos a soluciones de cloruro, que son los más perjudiciales para el acero inoxidable.

Esta investigación se realiza con el fin de concientizar a los profesionales de todas las ramas de ingeniería (proyectistas, contratistas, propietarios, consultores, etc.) de que existe una alternativa para diseñar o construir un proyecto que cumpla con todos los requisitos técnicos e incluso los supere, ya que las tuberías de polipropileno beta nucleado duran muchos años, a diferencia de una tubería de acero inoxidable que, a pesar de todo, se deteriorará.

\section{Corrosión en acero inoxidable}

El acero tiende a oxidarse cuando queda expuesto al ambiente, puesto que entra en contacto con el oxígeno y el vapor de agua presente en el aire, los cuales causan la formación de óxidos e hidróxidos de hierro en su superficie. Si no se frena, la oxidación penetra en todo el espesor de metal hasta corroerlo en su totalidad; por este motivo, existe una infinidad de estrategias para evitar que suceda.

El acero inoxidable se tiende a pasivar antes de permitir que la corrosión penetre su pared, de modo que se forma una fina película de óxido de cromo, la cual es más resistente a la corrosión; cuando esta película de óxido de cromo se elimina, vuelve a formarse al entrar el cromo en contacto con el oxígeno. Sin embargo, tal y como se ha comentado anteriormente, los aceros inoxidables siguen siendo vulnerables a la corrosión, que puede presentarse mediante distintos mecanismos:

\section{a. Corrosión intergranular}

Un tratamiento inadecuado (incluidos tanto las temperaturas usadas durante la fabricación del acero como los procedimientos de calentamiento durante las soldaduras) puede producir retículas con alta concentración en carbono, lo que promueve la corrosión intergranular. En estas condiciones, la superficie permite la oxidación del metal en condiciones corrosivas y reduce su vida útil en muchos ambientes relativamente ligeros.

\section{b. Corrosión galvánica}

La corrosión galvánica se produce de forma localizada y en distintas condiciones. Uno de 
los casos más comunes ocurre cuando dos metales distintos están en contacto directo (como es el caso de las uniones roscadas con válvulas de latón y tuberías de acero inoxidable o cobre); esta acción incrementa cuando se encuentran en ambientes húmedos o sumergidos en soluciones de electrolitos.

En un medio corrosivo, esta situación se agrava exponencialmente, lo que resulta en la disolución del electrodo anódico, mientras que el cátodo permanece inalterable. Los factores que influencian en mayor proporción el par galvánico son:

- Conductividad del fluido.

- Diferencia de potenciales de reducción de metales en contacto directo.

- Polarización.

- Áreas relativas del cátodo y ánodo en suma relación geométrica entre superficies de contacto.

\section{c. Aireación diferencial}

Una partícula semiincrustada en la superficie puede ser suficiente para eliminar la capa protectora de óxido de cromo. A pesar de que la partícula haya sido eliminada, su acción de atacar la superficie continúa, ya que se ha constituido una celda electrolítica duradera entre la porción de superficie anódica atacada y la vasta área catódica.

\section{d. Picadura}

Las soluciones de cloruros suelen causar corrosión por picadura en la superficie de contacto con acero inoxidable, que se ve prácticamente atacado mediante picaduras en las que se pueden desarrollar celdas electrolíticas.

Los cloruros ácidos, como el cloruro de hierro (III) y el cloruro de sodio, son dos de los compuestos que con mayor frecuencia promueven este tipo de corrosión, aunque cualquier cloruro en concentración apreciable puede causarla. Muchas veces los cloruros son el factor crítico en situaciones donde no se esperaría ver corrosión en el acero inoxidable.

\section{e. Corrosión por fatiga}

Aunque el usuario común no sea consciente de su existencia, la corrosión por fatiga es otro de los riesgos que suele promover y facilitar la presencia de corrosión en una tubería de acero inoxidable. Casi todos los metales y aleaciones pueden fallar al agrietarse a nivel microscópico en condiciones que impliquen la existencia de tensiones en un medio ligeramente oxidante. En este caso, también las soluciones de cloruros son uno de los medios más perjudiciales.

\section{f. Corrosión inducida por actividad microbio- lógica (MIC, por sus siglas en inglés)}

Debido a distintas evidencias, se ha empezado a sostener firmemente que muchos de los casos, en los que los procesos termodinámicos no explicaban la causa de la corrosión, pueden estar ocasionados por biofilms en la llamada Microbiology Influenced Corrosion (MIC). Actualmente, es objeto de múltiples investigaciones, desde distintos frentes.

Existen diversas causas para la propagación del material biológico en este tipo de corrosión; estas dependen de la bacteria responsable y de la morfología final de la superficie del metal. Por ejemplo, el $\mathrm{Fe}^{2+}$, ion comúnmente considerado un biocida, tiende a atraer bacterias reductoras de hierro que, por el contrario, lo usan en su metabolismo.

Una vez creado el biofilm, que se compone principalmente de agua, el ambiente se ve modificado radicalmente; así, el biofilm actúa como electrolito y puede llegar a alterar reacciones conocidas y controladas, o causar reacciones que no se desarrollarían sin presencia biológica.

Se puede observar que el principal causante de fallo en un acero inoxidable es la corrosión. Por su naturaleza, los polímeros son totalmente inmunes a dicho proceso.

Debemos tener en cuenta que en muchos proyectos realizados en el Perú se utilizan tuberías de acero inoxidable, ya sea para transportar fluidos como agua aséptica o fluidos que podrían ser corrosivos. Si bien las tuberías de acero inoxidable evitan la corrosión, estas tienen sus desventajas, como por ejemplo:

- El costo es muy alto.

- Presentan un peso elevado que podría sobrecargar la estructura de una edificación.

Como se ha mencionado, utilizar este tipo de tuberías presenta muchos inconvenientes; sin embargo, existen alternativas. El presente artículo propone el 
uso de las tuberías de polipropileno como una opción con múltiples ventajas, tales como:

- No se corroe.

- Es aséptica.

- Presenta menor peso.

- El costo es menor.

- Su instalación es más fácil y rápida.

El tema tratado en la presente investigación requiere mayor profundización, puesto que se trata de un proyecto que no tiene mucha aceptación en el país, ya que se siguen utilizando tuberías de acero inoxidable a pesar de que estas incurren en mayores costos de empleo y de la propia tubería. Las tuberías de acero inoxidable también son muy utilizadas en el sector minero para el transporte de agua corrosiva, pero estas tuberías de acero tienden a corroerse, y los tubos más apropiados para esa labor serían los de polipropileno.

El fin de este trabajo es incentivar el uso de tuberías de propileno en lugar de tuberías de acero inoxidable, ya que, si bien prima el uso de las primeras por su resistencia a la corrosión, estas son de alto costo y peso; además, su transporte es más difícil en comparación con las tuberías de polipropileno. Por otra parte, las tuberías de propileno se han ido desarrollando y mejorando con la ayuda de la ingeniería para que sus cualidades se asemejen a las características de las de acero inoxidable, de modo que puedan competir con estas.

\section{HIPÓTESIS}

Las tuberías de propileno son económicas y ambientalmente más óptimas que las tuberías de acero inoxidable.

\section{METODOLOGÍA}

¿Por qué elegir tuberías de polipropileno en vez de tuberías de acero inoxidable?

El sistema de tubería de polipropileno beta nucleado presenta ventajas con respecto a las propiedades fisicoquímicas de las tuberías de acero inoxidable.

\section{La evolución de las tuberías de polipropileno a tuberías de polipropileno beta nucleado}

La notable mejora de los materiales y el aprendizaje sobre sus propiedades mecánicas y físicas han originado un increíble y evidente avance en todos los campos. En fontanería, los metales fueron los materiales que más se emplearon hasta hace unas décadas debido a su resistencia mecánica. Sin embargo, con el paso del tiempo, estos materiales han presentado muchos inconvenientes (Serrano, 2017).

Según Serrano (2017), de las principales ventajas de las tuberías de polipropileno, que resuelven los problemas planteados en las instalaciones metálicas, se pueden destacar las siguientes:

- $100 \%$ de resistencia a la corrosión.

- Mínima conductividad térmica.

- Absorción a nivel acústico.

- Alta resistencia a los agentes químicos (material inerte ante soluciones de cloruros).

- Viabilidad.

- Reciclable en su totalidad, por lo que contribuye en la sostenibilidad del medio ambiente.

- Menor tiempo de instalación.

- Reducción del peso en la instalación.

Sin embargo, un aspecto importante que se debe tener en cuenta es el uso, cada vez mayor, de cloro en instalaciones de agua caliente para las desinfecciones de alto impacto en los materiales que muchas empresas se ven obligadas a ejecutar. De la necesidad de actuar frente a casos de termodegradación oxidativa ocasionada por este factor nació el sistema de tubería de polipropileno beta nucleado.

\section{Mejora de características termomecánicas}

La utilización del polímero RA7050 de Borealis, un polímero copolímero random con cristalinidad modificada y elevada resistencia a la temperatura (PP$\mathrm{R}$, tipo 4), ofrece una mejor característica mecánica con el paso de los años en instalaciones que transportan fluidos de alta temperatura; de esta manera garantiza la durabilidad a una mayor presión.

En la Figura 1 se observan las diferencias entre las curvas de referencia para las resistencias esperadas tanto del PP-R tipo 3 como del PP-R tipo 4, donde se observa que la curva del PP-R tipo 4 tiene una menor pendiente, lo que quiere decir que tiene un excelente comportamiento a elevadas temperaturas en comparación con el PP-R tipo 3.

Para profundizar en este aspecto, podemos comparar las gamas de tubería de polipropileno beta nucleado con las de tubería de polipropileno monocapa RP y tubería de fibra de polipropileno RP 


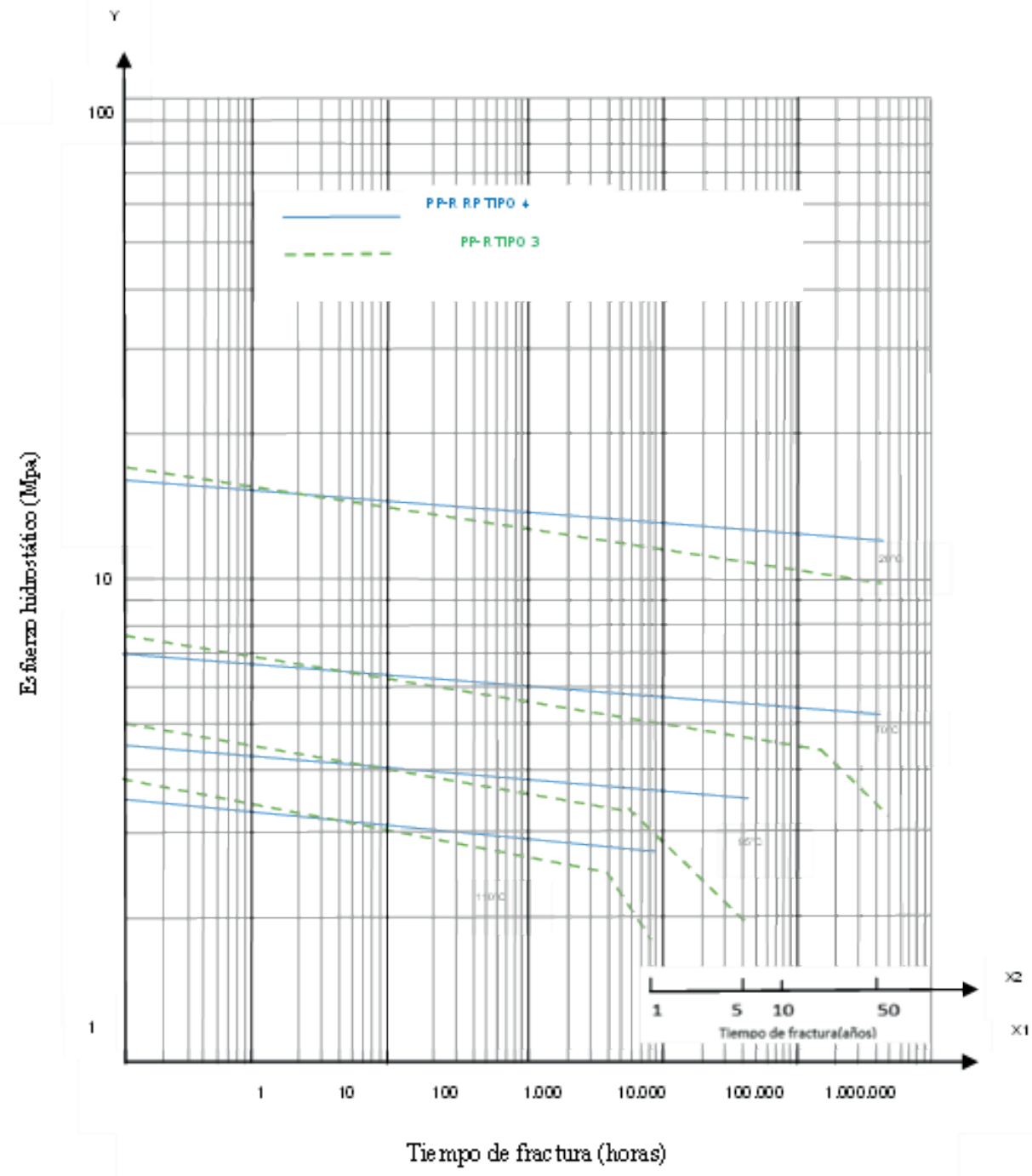

Figura 1. Curvas de referencia para la resistencia esperada del PP-R (Tipo 3) y PP-R (Tipo 4).

Fuente: Italsan, 2017.

con respecto a sus características de resistencia mecánica ante la temperatura y teniendo en cuenta su vida útil esperada. Así, se observa que la tubería de polipropileno beta nucleado presenta mayores prestaciones mecánicas debido a su SDR y aditivación, ya que la formulación de su materia prima es ligeramente superior a la del PP-R RP (Italsan, 2017).

\section{Resistencia a procesos de desinfección:}

Tal y como se establece en los protocolos de tratamiento de desinfección del Decreto Supremo N. ${ }^{\circ}$ 031-2010-SA (2010) así como del Real Decreto 865/2003 (2003), los compuestos químicos, como el cloro libre, deben usarse siempre por separado de los tratamientos por aumento de temperatura, como los choques térmicos.

Entre otras razones, esta especificación se hace porque los compuestos desinfectantes son de naturaleza oxidante y al aumentar la temperatura se convierten en agentes muy agresivos para la instalación. Este hecho ocurre tanto en distribuciones de tuberías de plástico como en tuberías de metales, incluyendo el cobre y el acero inoxidable AISI 316; también ocurre en otras partes de la instalación, como los acumuladores, intercambiadores y válvulas.

El sistema de tubería de polipropileno beta nucleado está formulado con una materia prima capaz de resistir una mayor concentración de cloro a mayor 
temperatura, teniendo en cuenta siempre que la distribución está sometida a presión. Obtuvo la clasificación de CL3 según ASTM F876 - 20B (American Society of Testing Materials, 2020) en los ensayos realizados por el prestigioso laboratorio Exova, acreditado para realizar tales operaciones. Según la norma ASTM F2023 (American Society of Testing Materials, 2015), las tuberías deben someterse a las siguientes condiciones:

- 4.3 ppm de hipoclorito de sodio

- $\mathrm{pH} 6.8$

- Temperaturas de $115^{\circ} \mathrm{C}, 105^{\circ} \mathrm{C}$ y $95^{\circ} \mathrm{C}$.

Con la altísima resistencia a la degradación termo-oxidativa mostrada en los resultados, se comprobó la superioridad del sistema de tuberías de polipropileno beta nucleado ante las demás gamas de PP-R y PP-R RP, por lo que quedó garantizado su uso en distribuciones a temperatura y presión elevadas con trasiego de fluidos con alto contenido de hipoclorito de sodio.

En una segunda instancia, en ensayos internos, se encontró que, en comparación con otras gamas de tuberías existentes en el mercado, las muestras de tubería de polipropileno beta nucleado ofrecieron valores de tiempos de inducción a la oxidación (OIt) un $40 \%$ superiores a las anteriores una vez expuestas las muestras a $4.5 \mathrm{ppm}$ de cloro libre, $95^{\circ} \mathrm{C}$ y 5 bar.

La relevancia del uso de un sistema de tubería de polipropileno beta nucleado radica en las facilidades que brinda desde un punto de vista técnico y económico, pues se trata de un material que manifiesta innovación al ser comparado con el acero inoxidable, gracias a su bajo costo y las bondades que brinda al realizar una instalación, puesto que contribuye a que esta se ejecute de manera rápida y sencilla. Cabe destacar que también significará un menor peso en la estructura civil que se ha instalado.

\section{RESULTADOS}

\section{Características físicas directamente relaciona- das con la eficiencia energética}

Al ser fabricado con PP-R, el sistema de tuberías de polipropileno tiene $100 \%$ garantizada la resistencia a la corrosión, tanto en el interior de la tubería como en su superficie, por lo que es totalmente inmune a los incrementos de conductividad eléctrica causados por la presencia de electrolitos o los cloruros. Este hecho presenta dos impactos que se producen en el cotejo con una instalación elaborada con tubería de acero inoxidable:

- El polipropileno no requiere ningún empleo de protección independientemente del medio circundante.

- El impacto es directamente proporcional a la eficiencia energética.

En este segundo apartado se desarrolla un análisis concreto, donde se demuestra que la disminución radical de probables depósitos e incrustaciones y la no existencia de corrosión permiten disminuir el consumo en los sistemas de bombeo durante toda la vida útil de la instalación, lo que reduce el consumo energético e incrementa la eficiencia de la instalación.

La pérdida de carga que se produce en las tuberías metálicas varía en función del estado de corrosión que existe en las mismas. En la Figura 2 se observan los resultados publicados por Rahmeyer (2009, como se citó en Italsan, 2015), donde se relaciona el aumento de las pérdidas de carga en una tubería metálica en función de su estado de corrosión.

\section{Contrastación de hipótesis:}

Con el fin de probar la eficiencia de un sistema de tubería de polipropileno beta nucleado frente a un sistema de tubería de acero inoxidable, se analizan los datos obtenidos en la Figura 2, donde se observa claramente la caída de carga de las tuberías metálicas debido a la corrosión a la que están expuestas. Además, al observar el estado de corrosión de ambos materiales, es posible notar la propiedad de total resistencia a la corrosión por parte del sistema de tubería de polipropileno beta nucleado y, a su vez, la limitada resistencia a la corrosión del acero inoxidable. De esta manera, queda en evidencia la optimización que significaría el uso del sistema de tubería de polipropileno beta nucleado en el punto específico de resistencia a la corrosión por parte del material.

También se cuenta con el conocimiento empírico de la carga estructural que ofrece el sistema de tubería de polipropileno beta nucleado, puesto que este material es mucho más liviano que un sistema de tubería de acero inoxidable, lo que permite contar con una estructura con menor peso y mayor factibilidad para su instalación (ver Tabla 1).

Cabe mencionar las ventajas económicas que conllevaría hacer uso de un sistema de tubería de polipropileno beta nucleado, ya que este material es mucho más económico en comparación con los 
- Corrosión severa

- Corrosión media

- Corrosión leve

- Tubería metálica nueva

- Tubería de polipropileno beta nucleada

.. Polinómica (Corrosión media)

Polinómica (Corrosión leve)

... Polinómica (Tubería metálica nueva)

........ Polinómica (Tubería de polipropileno beta nucleada)

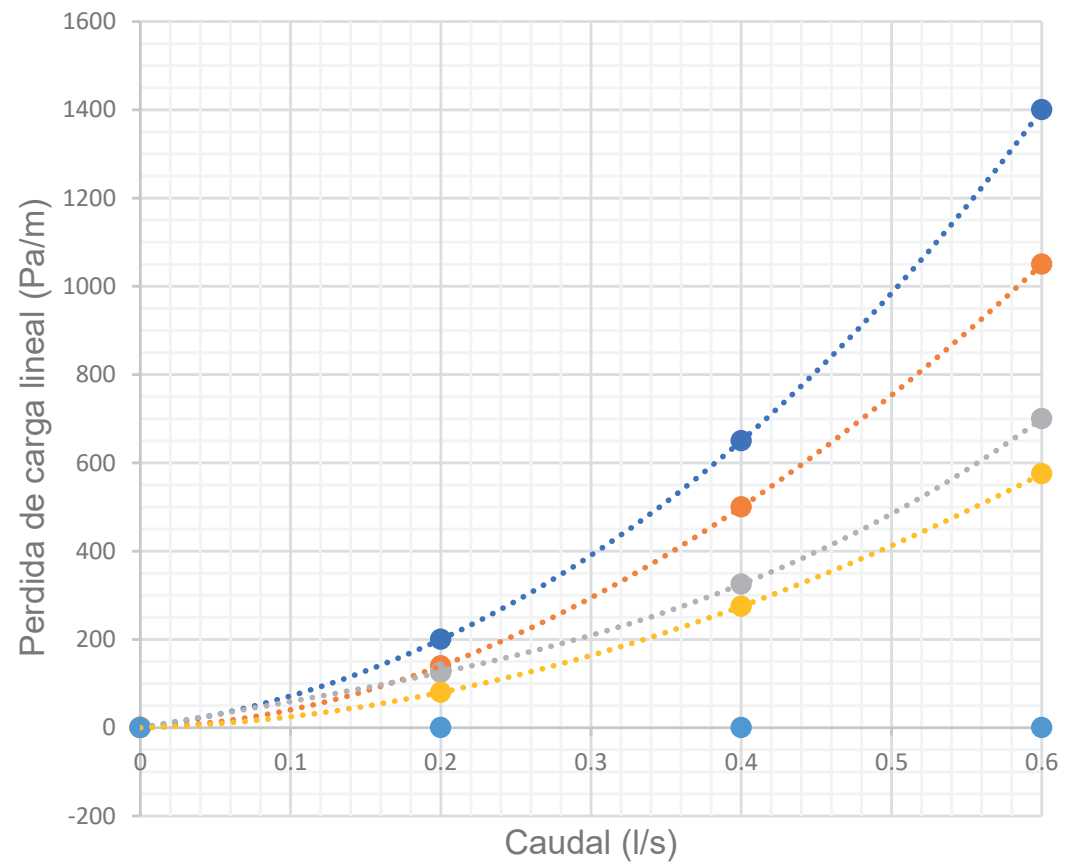

Figura. 2. Pérdidas de carga que se dan en las tuberías de metal versus estado de corrosión.

Fuente: Serrano (2017).

altos precios en los que incurre un sistema de tubería de acero inoxidable.

\section{DISCUSIÓN}

\section{Características físicas directamente relaciona- das con la eficiencia energética térmica}

a. Baja capacidad de transmisión de calor, dispersión térmica y condensación limitadas

La baja conductividad térmica de las tuberías de polipropileno $(\lambda=0,24 \mathrm{~W} / \mathrm{mK})$ puede provocar una reducción significativa de la temperatura del material, del diámetro y del espesor del aislamiento.
Los cálculos de espesor correspondientes se pueden realizar utilizando el software de cálculo de pérdidas del mercado internacional. El cálculo se basa en el coeficiente de conductividad térmica según UNE-EN ISO 12241:2010 (Organización Internacional de Normalización [ISO], 2010). Este procedimiento establece la pérdida de calor y la condensación de poros que se produce en la tubería (Tabla 2 ).

Existen 2 razones por las que se debe colocar aislamiento en la red de tuberías en instalaciones térmicas:

- Evitar la generación de condensaciones superficiales en la superficie de la tubería 
Tabla 1. Visión general del sistema tubería de polipropileno premium vs. acero inoxidable.

\begin{tabular}{|c|c|c|c|c|}
\hline Parámetro & Acero Inox. AISI 304 & Acero Inox. AISI 316 & $\begin{array}{c}\text { Sistema de tuberías de } \\
\text { polipropileno PP-R }\end{array}$ & $\begin{array}{l}\text { Sistema de tuberías de } \\
\text { polipropileno premium }\end{array}$ \\
\hline Resistencia a la corrosión & $\begin{array}{l}\text { Limitada a ciertos com- } \\
\text { puestos }\end{array}$ & $\begin{array}{c}\text { Superior a } 304, \text { limitada } \\
\text { a cloruros y otros }\end{array}$ & \multicolumn{2}{|c|}{ Total } \\
\hline Bioseguridad & \multicolumn{2}{|c|}{$\begin{array}{l}\text { Posibilidad de padecer MIC, fomentando también } \\
\text { tanto la adhesión como el crecimiento de biofilm }\end{array}$} & Alta & Superior \\
\hline $\begin{array}{l}\text { Ausencia de } \\
\text { incrustaciones }\end{array}$ & \multicolumn{2}{|c|}{ 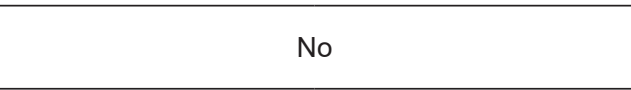 } & $\begin{array}{l}\text { Alta resistencia a } \\
\text { incrustaciones }\end{array}$ & $\begin{array}{l}\text { Superior resistencia a } \\
\text { incrustaciones }\end{array}$ \\
\hline $\begin{array}{l}\text { Idoneidad en zonas de } \\
\text { riesgo sísmico }\end{array}$ & \multicolumn{2}{|c|}{ No } & \multicolumn{2}{|c|}{ Sí } \\
\hline $\begin{array}{l}\text { Resistencia a corrientes } \\
\text { parásitas }\end{array}$ & \multicolumn{2}{|c|}{ Puede ser partícipe de su creación/propagación } & \multicolumn{2}{|c|}{ Total } \\
\hline $\begin{array}{l}\text { Compatible con agua } \\
\text { glicolada }\end{array}$ & \multicolumn{2}{|c|}{ Sí } & \multicolumn{2}{|c|}{ Sí } \\
\hline Conductividad térmica $(\lambda)$ & \multicolumn{2}{|c|}{$16.3 \mathrm{~W} /(\mathrm{mK})$} & $0.24 \mathrm{~W} /(\mathrm{mK})$ & $0.24 \mathrm{~W} /(\mathrm{mK})$ \\
\hline $\begin{array}{l}\text { Coeficiente dilatación } \\
\text { térmica lineal }\end{array}$ & \multicolumn{2}{|c|}{$0.017 \mathrm{~mm} /(\mathrm{mK})$} & \multicolumn{2}{|c|}{$0.04 \mathrm{~mm} /(\mathrm{mK})$} \\
\hline $\begin{array}{l}\text { Espesor necesario de } \\
\text { aislamiento (según RITE) }\end{array}$ & \multicolumn{2}{|c|}{ Según procedimiento simplificado del RITE } & \multicolumn{2}{|c|}{$\begin{array}{l}\text { Reducción del mismo según procedimiento } \\
\text { alternativo. }\end{array}$} \\
\hline Densidad & \multicolumn{2}{|c|}{$7.98 \mathrm{~g} / \mathrm{cm}^{3}$} & $0.90 \mathrm{~g} / \mathrm{cm}^{3}$ & $0.91 \mathrm{~g} / \mathrm{cm}^{3}$ \\
\hline $\begin{array}{l}\text { Velocidad máxima de } \\
\text { fluido (según CTE) }\end{array}$ & \multicolumn{2}{|c|}{$2.5 \mathrm{~m} / \mathrm{s}$} & \multicolumn{2}{|c|}{$3.5 \mathrm{~m} / \mathrm{s}$} \\
\hline Fonoabsorbencia & \multicolumn{2}{|c|}{ Limitada (conductor de vibraciones) } & \multicolumn{2}{|c|}{ Considerable } \\
\hline $\begin{array}{l}\text { Durabilidad estimada } \\
\text { normalizada }\end{array}$ & \multicolumn{2}{|c|}{ Sin datos asegurados } & 50 años & $\begin{array}{c}30 \text { años a } 4.3 \mathrm{ppm}, 60 \\
{ }^{\circ} \mathrm{C} \text { y } 5.5 \mathrm{bar}\end{array}$ \\
\hline $\begin{array}{l}\text { Tipología de unión } \\
\text { óptima }\end{array}$ & \multicolumn{2}{|c|}{ Soldadura con soplete } & \multicolumn{2}{|c|}{ Por termofusión } \\
\hline Suportación & \multicolumn{2}{|c|}{ Mediante abrazaderas/ perfil-abarcón } & \multicolumn{2}{|c|}{ Mediante abrazaderas } \\
\hline Montaje & \multicolumn{2}{|c|}{ Con alto requerimiento de especialización } & \multicolumn{2}{|c|}{ Sencillo, intuitivo y seguro } \\
\hline
\end{tabular}

Fuente: Elaboración propia. Datos obtenidos de Goodfellow (s.f.a; s.f.b), Acero Inoxidable AISI 304 y ASI 316.

Tabla 2. Ventajas en el uso del software de cálculo de pérdidas térmicas.

\begin{tabular}{|l|l|}
\hline Ventajas & PROGRAMA DE CÁLCULO DE PÉRDIDAS TÉRMICAS \\
\hline UNE-EN 12241 & Adecuación del espesor de aislamiento \\
\hline \multirow{3}{*}{} & Reducción del espacio \\
\cline { 2 - 2 } & Disminución del coste global de instalación \\
\cline { 2 - 2 } & Reducción de la demanda energética gracias a la eficiencia energética (garantía 4\% ) \\
\hline
\end{tabular}

Fuente: Serrano (2017).

- La pérdida total de calor de todas las tuberías no debe exceder el $4 \%$ de la potencia máxima de transporte, siguiendo lo establecido en el Reglamento de Instrucciones Térmicas de los Edificios (RITE) (Real Decreto 1027/2007, 2007).

\section{b. Disminución del riesgo de condensaciones}

Con base en el principio físico que indica que una masa de aire con temperatura y humedad relativas $X$ producirá condensaciones sobre la superficie del material siempre y cuando la temperatura superficial del aislamiento $\left(\mathrm{T}_{\mathrm{x}}\right)$ sea inferior a la temperatura de rocío del aire exterior en las condiciones de temperatura y humedad consideradas, se tiene que:

- se genera la condensación cuando $T_{\text {rocio }}$ $>T_{x}$

- no se genera la condensación cuando $\mathrm{T}_{\text {rocio }}<\mathrm{T}_{\mathrm{x}}$.

Para este caso, las condiciones menos favorables son la transferencia de fluido frío, temperatura ambiente alta y humedad relativa alta. 
El PP-R no es buen conductor del calor, o lo que es lo mismo, el valor de su conductividad térmica es muy bajo, por lo que el riesgo de condensaciones superficiales sobre la tubería disminuye y puede llegar a niveles muy bajos.

Además, en caso de existir condensación superficial, no existe la posibilidad de degradación por corrosión del material, puesto que el material es el mismo que el que se encuentra en el interior de las tuberías en contacto con el agua. Las tuberías metálicas poseen un coeficiente de conductividad térmica muy alto, por lo que el riesgo de condensación superficiales es muy superior al de las tuberías de PP-R.

\section{c. Disminución de las pérdidas térmicas}

Lo principal para una eficiente instalación es eludir el derroche de energía durante su producción y la siguiente repartición. La baja conductividad térmica del PP-R ayuda a mantener las temperaturas del fluido transportado, lo que resulta en mejoras gracias a la materia bruta, puesto que permite reducir en algunas ocasiones el espesor de aislamiento con respecto al considerado en el método simplificado del RITE. En estos casos, se reduce considerablemente el espacio necesario para la instalación, lo que representa un motivo por el cual elegir el sistema tubería de polipropileno en las actuaciones de reforma.

En el ordenamiento pseudoestacionario, la menor conductividad térmica del polipropileno, $\lambda=0.024 \mathrm{~W} /(\mathrm{mK})$, disminuye las alteraciones, lo que resulta favorable frente a una instalación con materiales metálicos; esto quiere decir que la dispersión térmica producida a lo largo de la instalación está más contenida, siendo innecesario producir tanto calor para suministrar ACS, calefacción y climatización hasta los puntos terminales (Italsan, s. f.).

Este suceso puede llegar a interpretarse como la disminución del consumo de la caldera de hasta un $10 \%$ en las instalaciones donde hay recirculación de fluido con temperatura a lo largo de las 24 horas del día y 365 días del año.

Como conclusión, la utilización de tuberías de PP-R y de una herramienta de cálculo de pérdidas térmicas garantizan pérdidas térmicas máximas de $4 \%$ con el espesor de aislamiento adecuado al material de la instalación.

\section{d. Mínimo grado de ruidos en la instalación}

Nuestro sistema de tuberías de polipropileno, gracias a su facultad de impregnación y apartamiento acústico, amortigua de forma notable los efectos sonoros en la instalación, ofreciendo bienestar acústico en las estancias.

\section{CONCLUSIONES}

\section{Reducción de tiempos de manipulación en la instalación}

El sistema de conexión de tuberías de polipropileno se basa en un acoplamiento molecular. Estos tipos de juntas no tienen precedentes en la industria de materiales poliméricos y ofrecen las siguientes ventajas:

- Tiempos de soldaduras más cortos (Norma DVS 2207-1), en comparación con las tuberías de acero inoxidable.

- Puesta en funcionamiento de la instalación inmediatamente después de la soldadura, sin tener que esperar para su enfriamiento como en el caso de tuberías metálicas.

Además, cabe resaltar que se puede prescindir de procesos de unión con intervención de llamas, es decir, los procesos para la conexión de tuberías de polipropileno son mucho más seguros que los de soldadura en acero inoxidable, los cuales exigen el uso de sopletes y protecciones específicas (Aristegui, 2017).

\section{Reducción del peso para manipulación}

Gracias a su densidad, que prácticamente tiene el mismo valor que la del agua, las tuberías realizadas en PP-R tienen un peso considerablemente menor que aquellas de acero inoxidable con mismo diámetro y longitud. Este hecho, además de disminuir el peso total de la instalación, supone una considerable disminución en los esfuerzos y, por lo tanto, en la dificultad de manipulación. Como las tuberías de PP-R son mucho más ligeras, pueden ser utilizadas por un mayor rango de usuarios y con una mayor comodidad.

\section{Simplicidad en unión}

Siempre que se mantengan los estándares de calidad durante el proceso, la termofusión es un proceso más sencillo que la soldadura de metales. En concreto, una mala aplicación de la llama durante la soldadura puede crear zonas propicias a la corrosión. 


\section{Bioseguridad}

Tanto la formación de óxido como la deposición de incrustaciones (como la cal) en la superficie de acero inoxidable aumenta su rugosidad, lo que favorece la acumulación de microorganismos (que pueden ser metabólicamente activos, a su vez, a los óxidos de metal) que pueden crear biocapa o biofilm, los cuales son agregados de material biológico que protegen a los microorganismos que en él se alojan y sirven también como medio para la evolución de estos. En este aspecto, el riesgo de legionelosis aumenta considerablemente. Como respuesta a estos hechos, el usuario debe aumentar la concentración de desinfectantes en el agua que, si bien podría destruir un máximo aproximado del $70 \%$ del biofilm, inicia o agrava procesos de corrosión.

Una vez acabadas las actuaciones de desinfección, los nuevos microorganismos (que siempre estarán presentes en el agua) tienen nuevos lechos creados en las partes más críticas de la instalación para depositarse de nuevo, creando así un ciclo que terminará con la reducción drástica de la vida útil de la instalación y con riesgos a nivel sanitario para la propiedad.

Gracias a su materia prima, el sistema tuberías de polipropileno es más resistente a incrustaciones y tiene una total inmunidad ante la corrosión.

Específicamente, la tubería de polipropileno beta nucleado ofrece una resistencia mayor a la termo-degradación oxidativa, por lo que la adherencia de biofilm, que es el factor clave a evitar para la proliferación de legionela, se ve reducida gracias al buen estado de la superficie de contacto con el fluido.

\section{AGRADECIMIENTO}

Josep María Ferrer Bruach, Doctor en Ciencias Químicas y responsable del desarrollo de nuevos productos en Italsan.

\section{REFERENCIAS BIBLIOGRÁFICAS}

[1] American Society of Testing Materials. (2015). ASTM F2023 - 15. Standard Test Method for Evaluating the Oxidative Resistance of Crosslinked Polyethylene (PEX) Pipe, Tubing and Systems to Hot Chlorinated Water. Recuperado de https://doi.org/10.1520/ F2023-15

[2] American Society of Testing Materials. (2020). ASTM F876 - 20B. Standard Specification for Crosslinked Polyethylene (PEX) Tubing. Recuperado de https://doi.org/10.1520/F0876$20 \mathrm{~B}$

[3] Aristegui Maquinaria. (23 diciembre de 2017). Norma DVS2207-1. Soldadura de tubería a testa. Recuperado de https://www.aristegui. info/norma-dvs2207-1-soldadura-de-tuberia-atesta/

[4] Asociación Española de Normalización y Certificación. (2010). Aislamiento térmico para equipos de edificación e instalaciones industriales. Método de cálculo. (ISO 12241:2008). Recuperado de https://www. aenor.com/normas-y-libros/buscador-denormas/une/?c=N0046295

[5] Concha, S. I. (2014). Diseño del plan de aseguramiento de la calidad del proceso de termofusión de tuberías de polipropileno. (Tesis de pregrado). Universidad Nacional del Callao, Callao.

[6] D. S. N. ${ }^{\circ}$ 031-2010-SA. Aprueban reglamento de la calidad del agua para consumo humano. Ministerio de Salud (2010). Recuperado de http://www.digesa.minsa.gob. pe/publicaciones/descargas/Reglamento_ Calidad_Agua.pdf

[7] Gea, E., Mezones, E., y Haro, L. (2012). Acciones de prevención y control de la legionelosis: un reto para la salud pública española. Revista Peruana de Medicina Experimental y Salud Publica, 29(2), 272-276.

[8] Goodfellow. (s. f.a). Acero Inoxidable - AlSI 304 - Catalogo en línea - Materiales en pequeñas cantidades para el diseño. Recuperado de http://www.goodfellow.com/S/AceroInoxidable-AISI-304.html

[9] Goodfellow. (s. f.b). Acero Inoxidable - AISI 316 - Catalogo en línea - Materiales en pequeñas cantidades para el diseño. Recuperado de http://www.goodfellow.com/S/AceroInoxidable-AISI-316.html

[10] Italsan. (s. f.). Sistema de tuberías de Polipropileno pre aislado para distribución de agua caliente y refrigerada. Recuperado de https://sustraiakgrupo.com/wp-content/ uploads/2018/09/Catalogo-NIRON-AIS-2013. pdf

[11] Italsan. (2015). Soluciones para la gestión eficiente de redes de distribución y evacuación de fluidos en Establecimientos Hoteleros y Alojamientos Turísticos. Recuperado de https:// 
italsan.com/media/files/documentos/catalogo_ hotelero_2015.pdf

[12] Italsan. (2017). La solución en salas de calderas - acumulación ACS. Recuperado de https://italsan.com/media/files/documentos/ catalogo_niron_premium_2017.pdf

[13] Real Decreto $865 / 2003$, de 4 de julio, por el que se establecen los criterios higiénico-sanitarios para la prevención y control de la legionelosis (2003). Boletín Oficial del Estado. Recuperado de https://www.boe.es/buscar/pdf/2003/BOEA-2003-14408-consolidado.pdf
[14] Real Decreto 1027/2007, de 20 de julio, por el que se aprueba el Reglamento de Instalaciones Térmicas en los Edificios (2007). Boletín Oficial del Estado. Recuperado de https://www.boe. es/eli/es/rd/2007/07/20/1027/dof/spa/pdf

[15] Serrano, E. (2017). Comparativa propiedades físico químicas: Sistemas termoplásticos vs tubería de acero. Hospitecnia, 1(9), 1-4. Recuperado de https://hospitecnia.com/sites/ default/files/158828495321588284953.pdf 


\section{Demonstrated Advantages and Considerations of a Beta Nucleated Polypropylene Piping System Versus a Stainless Steel Piping System}

\begin{abstract}
A polymer is a thermoplastic material that crystallizes in three forms: a (monoclinic), $\beta$ (trigonal) and $\gamma$ (orthorhombic). These forms have characteristics that provide specific physicochemical properties in the same formulation. The a crystalline form is found in higher proportion and is the most stable and simplest to produce. However, the raw material of the beta-nucleated polypropylene and PR (pressure resistant), monolayer pipes and PR fiber polypropylene pipes are processed to produce $\beta$ structures in ways where it would be easier to have a crystals, since this process favors the formation of a stable, fine hexagonal crystalline structure and a homogeneous size distribution. These attributes derive in an optimization of the mechanical performance of the system, in which a mixture of these forms ( $\alpha$ and $\beta$ ) will provide a very optimal thermo-mechanical property.
\end{abstract}

Keywords: PP-R (polypropylene random copolymer); transfer; biocidal product; austenitics; biofilm.

\section{INTRODUCTION}

American inventor John Hyatt created celluloid, a plastic material whose composition consists of a solution of carbohydrate from plants, cellulose and a mixture of ethyl alcohol and camphor; it is also known as "thermoplastic material" due to its ability to be molded and softened when exposed to heat (Concha, 2014).

Later, in 1907, Leo Baekeland invented Bakelite, which was described as the first thermoset or thermosetting plastic, that is, moldable, when melted. By 1930, polyethylene (PE), a thermoplastic resulting from the polymerization of ethylene gas under pressure and heat, was manufactured for the first time in England. Around 1950, the same process applied to propylene was used to create polypropylene. At present, this partially crystalline thermoplastic is classified as a polyolefin and is used extensively for multiple applications (Concha, 2014). Based on its properties, industrial applications are more associated with the use in the transport of acids and bases, even at high temperatures (Concha, 2014).

The beta nucleated polypropylene pipe system is one of the best choices for the manufacture of pipes intended for the transfer of fluids such as drinking water. The pressure and temperature working range of PP-R (polypropylene random copolymer) of the beta nucleated polypropylene piping system is fully compatible with treatments to prevent Legionella contamination during pipeline maintenance such as thermal shock.

1 Mechanical Electrical Engineer and $\mathrm{PhD}$ in Electrical Engineering from the Universidad Nacional de Ingeniería (Lima, Peru). Currently working as professor at the Universidad Nacional de Ingeniería (Lima, Peru).

ORCID: https://orcid.org/0000-0002-1755-6473

Corresponding author: ptoribio.consultor.ing@gmail.com

2 Degree in General Chemistry from Universidade da Coruña (A Coruña, España). Currently working as engineer at ITALSAN (Madrid, Spain). ORCID: https://orcid.org/0000-0002-3153-6284

E-mail: psantacruz@mundoecco.com

3 Metallurgical Engineer and $\mathrm{PhD}$ in Administration from Universidad Nacional de Ingeniería (Lima, Peru). Currently, Head of the School of Metallurgical Engineering and Head of the Graduate School of the Faculty of Mining Geological Engineering and Metallurgy of Universidad Nacional de Ingeniería (Lima, Peru).

ORCID: https://orcid.org/0000-0003-3748-120X

E-mail: albertolandauro@yahoo.es 
This means that Legionella prevention is under constant scrutiny and, consequently, undergoes periodic changes. This issue is more relevant in countries where there is a relationship between disease development and the country's industry. Such relationship is not apparent in other countries, either because of the lack of analysis or because Legionella contamination is not frequent. Indeed, continuous monitoring, new strategies and evaluation result in a good prevention of contracting diseases such as Legionellosis. It is a fact that the maintenance of equipment and the conditions of its environment are important; however, in urban areas, the disease is caused as a result of poor building design, inadequate construction and neglect of water quality control of the facilities (Gea, Mezones, \& Haro, 2012).

In summary, the PP-R of the beta nucleated polypropylene pipe system complies with Decreto Supremo No. 031-2010 (2010), which imposes regulations on the management of water quality for human consumption, in order to ensure its safety, avoid risks to human health and maintain population health.

$\mathrm{ClO}_{2}$ is not recommended for drinking water disinfection.

Historically, metals have always been the first choice for plumbing materials. However, as we have seen, technical polymers have been replacing them. Contrary to what its name specifies, stainless steel is not stainless. The reason it has this name is that, compared to normal steel, it can withstand much longer under the same working conditions before showing signs of wear. Not all stainless steels, however, have the same properties and, consequently, qualities.

Austenitic stainless steels are the most commonly used, of which the 300 series is the largest subgroup and, within this, AISI 304, followed by AISI 316, are the two most common types, whose high chromium and nickel content increase their resistance to corrosion. In addition, manganese, phosphorus, sulfur and silicon are also integrated into its chemical composition, which, in general, is iron (Goodfellow, n.d.a; Goodfellow, n.d.b). Some differences between the two alloys can be significant, depending on the structure, the most notable being the molybdenum content in AISI 316, which improves corrosion resistance with respect to AISI 304 in saline environments or environments exposed to chloride solutions, which are the most detrimental to stainless steel.

The aim of this research is to raise awareness among professionals in all branches of engineering (designers, contractors, owners, consultants, etc.) regarding the fact that there is an alternative to design or build a project that meets all the technical requirements and even exceeds them, since beta nucleated polypropylene pipes last for many years, unlike a stainless steel pipes that will nevertheless deteriorate.

\section{Corrosion in Stainless Steel}

Steel tends to rust when exposed to the environment, since it comes into contact with oxygen and water vapor present in the air, which cause the formation of iron oxides and hydroxides on its surface. If left unchecked, oxidation penetrates through the entire thickness of the metal until it corrodes it completely; for this reason, there are a myriad of strategies to prevent this from happening.

Stainless steel tends to undergo passivation before corrosion penetrates its wall, so that a thin film of chromium oxide forms, making it more resistant to corrosion. This film of chromium oxide, when removed, re-forms upon contact of the chromium with oxygen. Nonetheless, as discussed above, stainless steel is still vulnerable to corrosion, which can occur as a result of a number of different mechanisms:

\section{a. Intergranular Corrosion}

Inadequate treatment (including both the temperatures used during steelmaking and the heating procedures during welding) can produce high carbon-concentration lattices, which promote intergranular corrosion. Under these conditions, the surface allows the metal to oxidize and reduces its service life in many relatively light environments.

\section{b. Galvanic Corrosion}

Galvanic corrosion occurs locally and under different conditions. The most common is when two dissimilar metals are in direct contact (as in the case of threaded joints with brass valves and stainless steel or copper pipes); corrosion increases when exposed to humid environments or submerged in electrolyte solutions.

In a corrosive environment, this situation is exponentially aggravated, resulting in the dissolution of the anode, while the cathode remains unchanged. The factors that influence the galvanic couple to a greater extent are:

- Fluid connectivity 
- Difference of reduction potentials of metals in direct contact

- Polarization

- Relative areas of cathode and anode in geometric relation between contact surfaces

\section{c. Differential Aeration Corrosion}

A semi-embedded particle on the surface may be sufficient to remove the protective chromium oxide layer. Even after the particle has been removed, its attack on the surface continues, since a durable electrolytic cell has formed between the attacked portion of the anodic surface and the vast cathodic area.

\section{d. Pitting Corrosion}

Chloride solutions often cause pitting corrosion on the surface in contact with stainless steel, which is virtually attacked by pitting where electrolytic cells can develop.

Acid chlorides, such as iron (III) chloride and sodium chloride, are two of the most common compounds responsible for this type of corrosion, although any chloride in a significant concentration can cause pitting corrosion. Chlorides are often the critical factor in situations where stainless steel corrosion would not be expected.

\section{e. Fatigue Corrosion}

Although the average user may not be aware of its existence, fatigue corrosion is another factor that often leads to corrosion in stainless steel piping. Almost all metals and alloys can experience failure by cracking at the microscopic level under conditions involving stresses in a mildly oxidizing environment. Also in this case, chloride solutions are one of the most detrimental agents.

\section{f. Microbiology Influenced Corrosion (MIC)}

Based on the evidence, it has become firmly argued that many of the cases, where thermodynamic processes did not explain the cause of corrosion, may be caused by biofilms in the so-called Microbiology Influenced Corrosion (MIC). Currently, it is the subject of multiple studies on different fronts.

Depending on the bacteria responsible and the final morphology of the metal surface, there are several causes for the propagation of biological material in this type of corrosion. For example, $\mathrm{Fe}^{2+}$, an ion commonly considered a biocide, tends to attract iron-reducing bacteria which, on the other hand, use it in their metabolism.

Upon creation of the biofilm, which is composed mainly of water, the environment is radically modified; thus, the biofilm acts as an electrolyte and can alter known and controlled reactions, or cause reactions that would not take place without the biological presence.

Clearly, corrosion is the main cause of failure in stainless steel. Polymers, on the other hand, are completely immune to this process due to their nature.

Many projects in Peru use stainless steel pipes, either to transport fluids such as aseptic water or other fluids that could be corrosive. Although stainless steel pipes prevent corrosion, there are some disadvantages, such as:

- They are very expensive.

- They are very heavy, which can overload the structure of a building.

As stated above, the use of this type of piping presents many disadvantages, but there are alternatives. In this paper, we propose the use of polypropylene pipes as an option with multiple advantages, such as:

- It does not corrode.

- It is asceptic.

- It is lighter.

- It is less expensive.

- Its installation is easier and faster.

Further research is required on the research topic, as it is not very popular in the country; stainless steel pipes are still being used, despite the fact that they are more expensive. Stainless steel pipes are also widely used in the mining sector for the transport of corrosive water, even though these steel pipes tend to corrode, and polypropylene pipes would be the most appropriate for this task.

The purpose of this study is to encourage the use of propylene pipes instead of stainless steel pipes, since although the use of the former is more important due to their resistance to corrosion, they are expensive and heavy; also, they are more difficult to transport compared to polypropylene pipes. Moreover, propylene pipes have been developed and 
improved with the help of engineering so that their qualities are similar to those of stainless steel pipes and can compete with the latter.

\section{HYPOTHESIS}

Propylene pipes are more cost-effective and environmentally friendly than stainless steel pipes.

\section{METHODOLOGY}

Why choose polypropylene pipes instead of stainless steel pipes?

The beta nucleated polypropylene pipe system has advantages over the physicochemical properties of stainless steel pipes.

\section{The evolution from polypropylene pipes to beta nucleated polypropylene pipes}

The remarkable improvement of materials and learning about their mechanical and physical properties has led to tremendous and obvious progress in all fields. In plumbing, metals were the most commonly used materials until a few decades ago due to their mechanical strength. However, as time went by, these materials have presented many drawbacks (Serrano, 2017).

According to Serrano (2017), the following advantages of polypropylene pipes, which solve the problems posed in metallic structures, can be highlighted:

- $100 \%$ corrosion resistance

- Minimum thermal conductivity

- Acoustic absorption

- High resistance to chemical agents (inert material to chloride solutions)

- Viability

- Fully recyclable, contributes to environmental sustainability

- Shorter installation time

- Reduced structure weight.

A major consideration, however, is the increasing use of chlorine in hot water distribution systems for high-impact disinfection of materials that many companies are forced to carry out. The beta nucleated polypropylene pipe system resulted from the need to deal with cases of thermo-oxidative degradation caused by this factor.

\section{Improvement of Thermo-Mechanical Characteristics}

Borealis RA7050 polymer, a random copolymer polymer with modified crystallinity and high temperature resistance (PP-R, type 4), offers improved mechanical characteristics over the years in structures that transport high-temperature fluids, thus ensuring durability at higher pressure.

Figure 1 shows the differences between the reference curves for the expected strength of both PP-R type 3 and PP-R type 4. As can be observed, PP-R type 4 has a lower slope, which means that it has an excellent performance at high temperatures compared to PP-R type 3.

For a deeper analysis of this aspect, we can compare the ranges of beta nucleated polypropylene pipes with those of RP single-layer polypropylene pipes and RP polypropylene fiber pipes with respect to their mechanical strength characteristics at high temperatures, considering their expected service life. Thus, the beta nucleated polypropylene pipe has a higher mechanical performance due to its SDR and additivation, since the formulation of its raw material is slightly higher than that of PP-R RP (Italsan, 2017).

\section{Resistance to Disinfection Processes}

As established in the disinfection treatment protocols of Decreto Supremo No. 031-2010-SA (2010) as well as Real Decreto 865/2003 (2003), chemical compounds, such as free chlorine, should always be used separately from treatments involving temperature increase, such as thermal shocks.

This is specified, among other reasons, because disinfectant compounds have an oxidizing nature and become very aggressive agents as temperature increases. This happens both in plastic or metal pipe systems, including copper and stainless steel AISI 316; it also occurs in other parts of the system, such as accumulators, heat exchangers and valves.

The beta nucleated polypropylene piping system is made of a raw material capable of enduring a higher concentration of chlorine at a higher temperature, bearing in mind that the system is always under pressure. It was rated CL3 according to ASTM F876 20B (American Society of Testing Materials, 2020 ) in tests carried out by the prestigious Exova laboratory, accredited to perform such operations. According to ASTM F2023 (American Society of Testing Materials, 2015), pipes must be subjected to the following specifications:

\section{- 4.3 ppm sodium hypochlorite}




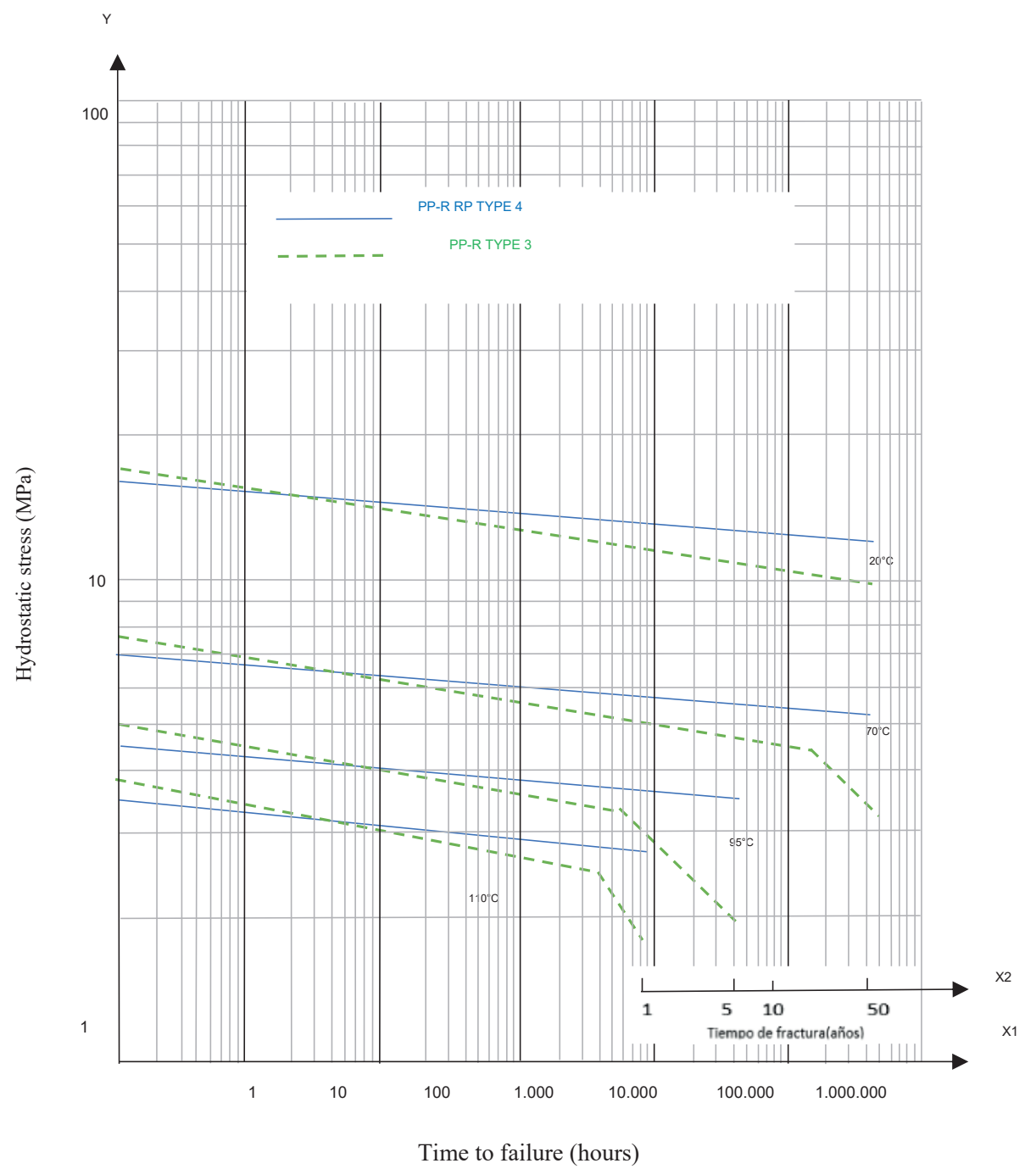

Figure 1. Reference curves for the expected strength of PP-R (Type 3) and PP-R (Type 4).

Source: Italsan, 2017.

- $\mathrm{pH} 6.8$

- Temperatures of $115^{\circ} \mathrm{C}, 105^{\circ} \mathrm{C}$ and $95^{\circ} \mathrm{C}$.

The very high resistance to thermo-oxidative degradation shown in the results proved the superiority of the beta nucleated polypropylene pipe system over PP-R and PP-R RP pipes; therefore, its use in high temperature and pressure piping systems with transfer of fluids with high sodium hypochlorite content was certified.

Internal tests showed that, compared to other pipe types on the market, the beta nucleated polypropylene pipe samples had $40 \%$ higher oxidation induction times (Olt) when exposed to $4.5 \mathrm{ppm}$ of free chlorine, $95^{\circ} \mathrm{C}$ and 5 bar pressure.
The importance of using a beta nucleated polypropylene piping system lies on the technical and economic advantages it offers, since it is an innovative material when compared to stainless steel, due to its low cost and benefits when implementing a system, as it allows for a quick and easy installation. It should be noted that it will also mean less weight added to the civil structure being installed.

\section{RESULTS}

\section{Physical Characteristics Directly Related to Energy Efficiency}

Being manufactured with PP-R, polypropylene piping systems are $100 \%$ corrosion resistant, both inside the pipe and on its surface, making it totally 
immune to increases in electrical conductivity caused by the presence of electrolytes or chlorides. In comparison with a stainless steel pipe system, polypropylene:

- does not require any protective measures regardless of the surrounding environment,

- its impact is directly proportional to the energy efficiency.

A specific analysis is performed in this second section, evidencing that the radical reduction of probable deposits and fouling and the absence of corrosion reduce consumption in the pumping systems during the entire service life of the system, thus reducing energy consumption and increasing the efficiency of the system.
Head loss in steel piping varies depending on the degree of corrosion of the pipes. Figure 2 shows the results obtained by Rahmeyer (2009, as cited in Italsan, 2015), where the increase in head losses in a steel pipe is related to its corrosion state.

\section{HYPOTHESIS TESTING}

An analysis of the data shown in Figure 2 is made in order to test the efficiency of a beta nucleated polypropylene pipe system versus a stainless steel pipe system; the head drop of the steel pipes due to corrosion is clearly observed. Upon observing the corrosion state of both materials, it is noticeable the total corrosion resistance property of the beta nucleated polypropylene pipe system, as well as the limited corrosion resistance of the stainless
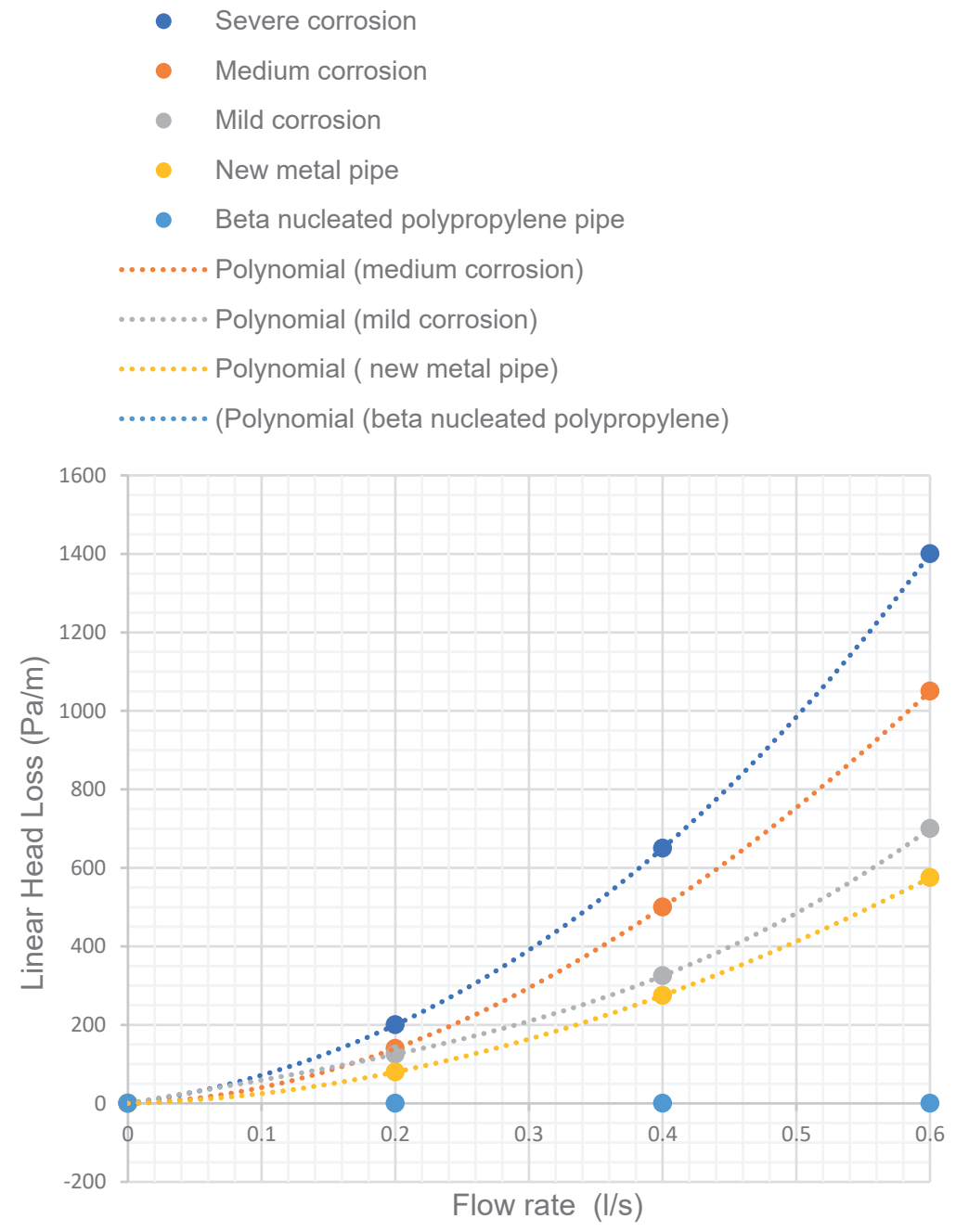

Figure. 2. Head losses in steel pipes vs. corrosion state. Source: Serrano (2017). 
steel. On this basis, it is evidenced that the use of beta nucleated polypropylene piping systems would represent an optimization regarding corrosion resistance of the material.

There is also empirical knowledge concerning the structural load of the beta nucleated polypropylene pipe system, as this material is much lighter than a stainless steel pipe system, which allows for a lighter weight structure and easier installation (see Table 1).

Attention should be drawn to the economic advantages of using a beta nucleated polypropylene pipe system, since this material is much more economical compared to the high prices of a stainless steel pipe system.

\section{DISCUSSION}

\section{Physical Characteristics Directly Related to Thermal Energy Efficiency}

a. Low heat transfer capacity, limited heat dissipation and condensation

The low heat conductivity of polypropylene pipes $(\lambda=0.24 \mathrm{~W} / \mathrm{mK})$ can lead to a significant reduction in material temperature, diameter and insulation thickness.

Thickness can be calculated using the loss calculation software on the international market. The calculation is based on the thermal conductivity coefficient according to UNE-EN ISO

Table 1. Premium Polypropylene vs. Stainless Steel Piping System Overview.

\begin{tabular}{|c|c|c|c|c|}
\hline Parameter & $\begin{array}{l}\text { AISI } 304 \text { Stainless } \\
\text { Steel }\end{array}$ & $\begin{array}{c}\text { AISI } 316 \text { Stainless } \\
\text { Steel }\end{array}$ & $\begin{array}{l}\text { PP-R Polypropylene } \\
\text { Piping System }\end{array}$ & $\begin{array}{l}\text { Premium Polypropyle- } \\
\text { ne Piping System }\end{array}$ \\
\hline Corrosion resistance & $\begin{array}{l}\text { Limited to certain com- } \\
\text { pounds }\end{array}$ & $\begin{array}{l}\text { Above } 304 \text {, limited to } \\
\text { chlorides and others }\end{array}$ & \multicolumn{2}{|c|}{ Total } \\
\hline Biosafety & \multicolumn{2}{|c|}{$\begin{array}{c}\text { Potential for MIC, also promoting both adhesion } \\
\text { and biofilm growth }\end{array}$} & High & Superior \\
\hline Absence of fouling & \multicolumn{2}{|c|}{ No } & High fouling resistance & $\begin{array}{l}\text { Superior fouling resis- } \\
\text { tance }\end{array}$ \\
\hline $\begin{array}{l}\text { Suitability in seismic risk } \\
\text { areas }\end{array}$ & \multicolumn{2}{|c|}{ No } & \multicolumn{2}{|c|}{ Yes } \\
\hline $\begin{array}{l}\text { Resistance to stray } \\
\text { currents }\end{array}$ & \multicolumn{2}{|c|}{ Can participate in its planning/difussion } & \multicolumn{2}{|c|}{ Total } \\
\hline $\begin{array}{l}\text { Compatible with glycol } \\
\text { water }\end{array}$ & \multicolumn{2}{|c|}{ Yes } & \multicolumn{2}{|c|}{ Yes } \\
\hline Thermal conductivity $(\lambda)$ & \multicolumn{2}{|c|}{$16.3 \mathrm{~W} /(\mathrm{mK})$} & $0.24 \mathrm{~W} /(\mathrm{mK})$ & $0.24 \mathrm{~W} /(\mathrm{mK})$ \\
\hline $\begin{array}{l}\text { Coefficient of linear ther- } \\
\text { mal expansion }\end{array}$ & \multicolumn{2}{|c|}{$0.017 \mathrm{~mm} /(\mathrm{mK})$} & \multicolumn{2}{|c|}{$0.04 \mathrm{~mm} /(\mathrm{mK})$} \\
\hline $\begin{array}{l}\text { Necessary insulation } \\
\text { thickness (according to } \\
\text { RITE }^{4} \text { ) }\end{array}$ & \multicolumn{2}{|c|}{ According to the RITE simplified procedure } & \multicolumn{2}{|c|}{ Reduction according to alternative procedure } \\
\hline Density & \multicolumn{2}{|c|}{$7.98 \mathrm{~g} / \mathrm{cm}^{3}$} & $0.90 \mathrm{~g} / \mathrm{cm}^{3}$ & $0.91 \mathrm{~g} / \mathrm{cm}^{3}$ \\
\hline $\begin{array}{l}\text { Maximum fluid velocity } \\
\text { (according to CTE }{ }^{5} \text { ) }\end{array}$ & \multicolumn{2}{|c|}{$2.5 \mathrm{~m} / \mathrm{s}$} & \multicolumn{2}{|c|}{$3.5 \mathrm{~m} / \mathrm{s}$} \\
\hline Sound absorption & \multicolumn{2}{|c|}{ Limited (vibration conductor) } & \multicolumn{2}{|c|}{ Significant } \\
\hline $\begin{array}{l}\text { Estimated normalized } \\
\text { service life }\end{array}$ & \multicolumn{2}{|c|}{ Uncertain data } & 50 years & $\begin{array}{l}30 \text { years at } 4.3 \text { ppm, } 60 \\
{ }^{\circ} \mathrm{C} \text { and } 5.5 \text { bar }\end{array}$ \\
\hline Optimal joint type & \multicolumn{2}{|c|}{ Torch welding } & \multicolumn{2}{|c|}{ Thermofusion welding } \\
\hline Support & \multicolumn{2}{|c|}{ Clamps/Profile bars } & \multicolumn{2}{|c|}{ Clamps } \\
\hline Mounting & \multicolumn{2}{|c|}{ High specialization requirements } & \multicolumn{2}{|c|}{ Simple, intuitive and safe } \\
\hline
\end{tabular}

Source: Prepared by the authors based on data from Goodfellow (n.d.a; n.d.b), AISI 304 and ASI 316 stainless steel.

4 Spanish standard for thermal installation in buildings.

5 Spanish technical building code. 
12241:2010 (Organización Internacional de Normalización [ISO], 2010). This procedure determines the heat loss and pore condensation that occurs in the pipe (Table 2).

There are two reasons why insulation should be placed in the pipe network of thermal installations:

- To prevent the generation of surface condensation on the pipe surface.

- The total heat loss of all pipes should not exceed $4 \%$ of the maximum transport power, as established in the Regulation of Thermal Instructions for Buildings (RITE) (Royal Decree 1027/2007, 2007).

\section{b. Reduction of condensation risk}

Based on the physical principle that indicates that a mass of air with relative temperature and humidity $\mathrm{X}$ will produce condensation on the surface of the material as long as the surface temperature of the insulation $\left(T_{x}\right)$ is lower than the dew temperature of the outside air under the temperature and humidity conditions considered, it follows that:

- condensation occurs when Tdp > Tx

- condensation does not occur when Tdp $<$ Tx.

For this case, the least favorable conditions are cold fluid transfer, high ambient temperature and high relative humidity.

PP-R is not a good conductor of heat, and its thermal conductivity value is very low, so the risk of surface condensation on the pipe decreases and can reach very low levels.

In addition, in the event of surface condensation, there is no possibility of degradation by corrosion of the material, since the material is the same as that found inside the pipes in contact with water. Steel pipes have a very high thermal conductivity coefficient; therefore, the risk of surface condensation is much higher than that of PP-R pipes.

\section{c. Reduction of thermal losses}

The main issue for an efficient system is to avoid wasting energy during its production and subsequent distribution. The low thermal conductivity of PP-R helps maintaining the temperatures of the transported fluid, resulting in improvements due to the raw material, since it sometimes allows reducing the insulation thickness with regard to that considered in the simplified method of the RITE. In these cases, the space required for pipes is considerably reduced, and this represents a reason for choosing the polypropylene pipe system in renovation works.

In the pseudo-stationary arrangement, the lower thermal conductivity of polypropylene, $\lambda=0.024$ $\mathrm{W} /(\mathrm{mK})$, reduces the disturbances, which is favorable compared to systems with metallic materials. The thermal dispersion produced along the system is more contained; therefore, producing so much heat to supply DHW, heating and air conditioning up to the terminal points is unnecessary (Italsan, n. d.).

Such an event can be interpreted as a decrease in boiler consumption of up to $10 \%$ in systems where there is recirculation of fluid with temperature 24 hours a day and 365 days a year.

In conclusion, the use of PP-R pipes and a thermal loss calculation tool guarantee maximum thermal losses of $4 \%$ with the insulation thickness appropriate to the material of the system.

\section{d. Minimum noise level in the system}

Owing to its acoustic impregnation and isolation properties, our polypropylene piping system significantly dampens the sound effects in the installation, providing acoustic comfort in all rooms.

Table 2. Advantages of Using Heat Loss Calculation Software.

\begin{tabular}{|l|l|}
\hline Advantages & HEAT LOSS CALCULATION SOFTWARE \\
\hline UNE-EN 12241 & Adequacy of insulation thickness \\
\hline \multirow{5}{*}{} & Space reduction \\
\cline { 2 - 2 } & Reduction of the overall installation cost \\
\cline { 2 - 2 } & Reduction of energy demand thanks to energy efficiency (4\% guarantee) \\
\hline
\end{tabular}

Source: Serrano (2017). 


\section{CONCLUSIONS}

\section{Reduced Handling Times}

The polypropylene pipe connection system is based on a molecular coupling. These types of joints are unprecedented in the polymeric materials industry and offer the following advantages:

- Shorter welding times (DVS Standard 22071) compared to stainless steel pipes

- Immediate start-up of the system after welding, without having to wait for cooling as in the case of metallic pipes.

Note that joining processes involving flames can be avoided, i.e., the processes for connecting polypropylene pipes are much safer than stainless steel welding, which requires the use of torches and specific protections (Aristegui, 2017).

\section{Reduced Weight for Handling}

Owing to its density, which is virtually the same as that of water, pipes made of PP-R have a considerably lower weight than those made of stainless steel with the same diameter and length. This fact, in addition to reducing the total weight of the system, means a considerable reduction in stresses and, therefore, in the difficulty of handling. As PP-R pipes are much lighter, a wider range of users can comfortably use them.

\section{Joining Simplicity}

Provided that quality standards are maintained during the process, thermofusion is much simpler than metal welding. Specifically, poor flame application during welding can create corrosion-prone areas.

\section{Biosafety}

Both the formation of rust and fouling (such as scale) on the stainless steel surface increases its roughness, which favors the accumulation of microorganisms (which in turn may be metabolically active to the metal oxides) that can create biofilms, which are aggregates of biological material that protect the microorganisms that lodge therein and also serve as a medium for their evolution. Risk of legionellosis increases considerably in this respect. Consequently, users have to increase the concentration of disinfectants in the water which, although it may destroy a maximum of approximately $70 \%$ of the biofilm, it initiates or aggravates corrosion processes.
Once the disinfection actions are finished, the new microorganisms (always present in the water) have new settlements in the most critical parts of the installation to be deposited again, thus creating a cycle that will lead to a drastic reduction of the service life of the system and to sanitary risks.

Owing to its composition, the polypropylene piping system is more resistant to fouling and is completely immune to corrosion.

Specifically, the beta nucleated polypropylene pipe offers a higher resistance to thermo-oxidative degradation, so that the adherence of biofilm, which must be avoided to prevent the proliferation of Legionella, is reduced thanks to the good condition of the surface in contact with the fluid.

\section{ACKNOWLEDGEMENT}

To Josep María Ferrer Bruach, PhD in Chemical Science and head of new product development at Italsan.

\section{REFERENCIAS BIBLIOGRÁFICAS}

[1] American Society of Testing Materials. (2015). ASTM F2023 - 15. Standard Test Method for Evaluating the Oxidative Resistance of Crosslinked Polyethylene (PEX) Pipe, Tubing and Systems to Hot Chlorinated Water. Retrieved from https://doi.org/10.1520/F202315

[2] American Society of Testing Materials. (2020). ASTM F876 - 20B. Standard Specification for Crosslinked Polyethylene (PEX) Tubing. Retrieved from https://doi.org/10.1520/F0876$20 \mathrm{~B}$

[3] Aristegui Maquinaria. (December 23, 2017). Norma DVS2207-1. Soldadura de tubería a testa. Retrieved from https://www.aristegui. info/norma-dvs2207-1-soldadura-de-tuberia-atesta/

[4] Asociación Española de Normalización y Certificación. (2010). Aislamiento térmico para equipos de edificación e instalaciones industriales. Método de cálculo. (ISO 12241:2008). Retrieved from https://www. aenor.com/normas-y-libros/buscador-denormas/une/?c=N0046295

[5] Concha, S. I. (2014). Diseño del plan de aseguramiento de la calidad del proceso de termofusión de tuberías de polipropileno. 
(Tesis de pregrado). Universidad Nacional del Callao, Callao.

[6] D. S. N. ${ }^{\circ}$ 031-2010-SA. Aprueban reglamento de la calidad del agua para consumo humano. Ministerio de Salud (2010). Retrieved from http:// www.digesa.minsa.gob.pe/publicaciones/ descargas/Reglamento_Calidad_Agua.pdf

[7] Gea, E., Mezones, E., \& Haro, L. (2012). Acciones de prevención y control de la legionelosis: un reto para la salud pública española. Revista Peruana de Medicina Experimental y Salud Publica, 29(2), 272-276.

[8] Goodfellow. (n. d.a). Acero Inoxidable AISI 304 - Catalogo en línea - Materiales en pequeñas cantidades para el diseño. Retrieved from http://www.goodfellow.com/S/AceroInoxidable-AISI-304.html

[9] Goodfellow. (n. d.b). Acero Inoxidable AISI 316 - Catalogo en línea - Materiales en pequeñas cantidades para el diseño. Retrieved from http://www.goodfellow.com/S/AceroInoxidable-AISI-316.html

[10] Italsan. (n. d.). Sistema de tuberías de Polipropileno pre aislado para distribución de agua caliente y refrigerada. Retrieved from https://sustraiakgrupo.com/wp-content/ uploads/2018/09/Catalogo-NIRON-AIS-2013. pdf
[11] Italsan. (2015). Soluciones para la gestión eficiente de redes de distribución y evacuación de fluidos en Establecimientos Hoteleros y Alojamientos Turísticos. Retrieved from https:// italsan.com/media/files/documentos/catalogo_ hotelero_2015.pdf

[12] Italsan. (2017). La solución en salas de calderas - acumulación ACS. Retrieved from https://italsan.com/media/files/documentos/ catalogo_niron_premium_2017.pdf

[13] Real Decreto $865 / 2003$, de 4 de julio, por el que se establecen los criterios higiénico-sanitarios para la prevención y control de la legionelosis (2003). Boletín Oficial del Estado. Retrieved from https://www.boe.es/buscar/pdf/2003/ BOE-A-2003-14408-consolidado.pdf

[14] Real Decreto 1027/2007, de 20 de julio, por el que se aprueba el Reglamento de Instalaciones Térmicas en los Edificios (2007). Boletín Oficial del Estado. Retrieved from https://www.boe.es/ eli/es/rd/2007/07/20/1027/dof/spa/pdf

[15] Serrano, E. (2017). Comparativa propiedades físico químicas: Sistemas termoplásticos vs tubería de acero. Hospitecnia, 1(9), 1-4. Retrieved from https://hospitecnia.com/sites/ default/files/158828495321588284953.pdf 